\title{
Dynamic positioning accuracy of dual-axis drive mechanism of satellite antenna with multi-clearance coupling
}

\author{
Chaoqun Qi ${ }^{1}$, Huibo Zhang ${ }^{1}$, Bindi You ${ }^{2}$, Jizhuang Fan ${ }^{3}$ and Shijie Dai ${ }^{1}$ \\ ${ }^{1}$ Hebei University of Technology, Hebei Key Laboratory of Robot Sensing and Human-Robot Interaction, Tianjin, 300130, PR China \\ ${ }^{2}$ School of Naval Architecture and Ocean Engineering, Harbin Institute of Technology at Weihai, Weihai 264209, Shandong, PR China \\ ${ }^{3}$ State Key Laboratory of Robotics and System, Harbin Institute of Technology, Harbin 150001, Heilongjiang, PR China
}

\begin{abstract}
The dual-axis drive mechanism of satellite antenna as a typical multi-joint aerospace mechanism consists of azimuth axis and pitch axis. The multi-clearance contained within those drive joint cause contact and impact in joint, and consequently affect the dynamic positioning accuracy. A nonlinear dynamic model of planetary gears with multiple clearances couplings is proposed to investigate the influence mechanism of clearances on the dynamic performances. The nonlinear factors such as radial clearance, backlash and time-varying meshing stiffness of the bearing are considered in the model. And the dynamic model of double-axis driving mechanism of satellite antenna with multi-clearance coupling is established. Then positional and rotational speed error curves of antenna reflector at different rotational speed are respectively analyzed. The numerical simulation results show that the influence of multi-clearance coupling on the dynamic positioning accuracy of double-axis drive mechanism of satellite antenna is remarkable.
\end{abstract}

\section{Introduction}

It is known that planetary gears are widely used in space mechanism. However, clearances in planetary gears are inevitable due to manufacturing, assembly errors, and wear. Those clearances cause contact and impact between gear sets, and consequently affect the control accuracy $[1,2]$. So the research on the vibration characteristics of planetary gear system are receiving more and more attention to obtain the high precision and high reliability of space mechanism.

From the modeling methodology point of view, several different methods have been developed. Kahraman [3-5] has presented a series of nonlinear time-varying dynamic models of planetary gears. Filipe [6] presented a new formulation to model spatial revolute joints with radial and axial clearances. Parker [7-9] analyzed the vibration mode of spur gear system using finite element method. Pan Bo [10] established a planetary gear dynamics model for the space manipulator joint considering the nonlinear factors. However, the current model ignores the effect of multi-clearance coupling on the vibration characteristics of planetary gear system. Multiple clearances are main factors that cause the performance and reliability decline of complicated mechanical systems. Those clearances cause contact and impact in joint, and consequently affect the control accuracy.
In this paper, a nonlinear dynamic model of planetary gears with multi-clearance couplings is proposed. Then the dynamic model of double-axis driving mechanism of satellite antenna is established. Finally, the effects of multi-clearance coupling on positional and rotational speed error curves of antenna reflector at different rotational speed are studied and discussed respectively by numerical simulation.

\section{Dynamic model of system with multi-clearance coupling}

\subsection{Dynamic Model of Planetary Gear Drive Joint with multi-clearance coupling}

$2 \mathrm{~K}-\mathrm{H}$ planetary gear reducer as a typical joint system is the study object of current study. The system is composed of four parts: sun gear (s), ring gear (r), planet carrier (c), and a certain number of planet gears (p). It is known that a radial clearance exists in bearing, and a backlash occurs between the teeth. So that vibrations will occur in clearance during the course of movement, then the radial vibration at bearing lead to the change of gear's actual center distance, then the backlash will dynamically changed. The appearance of dynamic backlash will affect torsional vibration and even the whole vibration characteristics of gear system. Therefore, the dynamics 
model of planetary gear transmission with multi clearance coupling is established as shown in Figure 1.

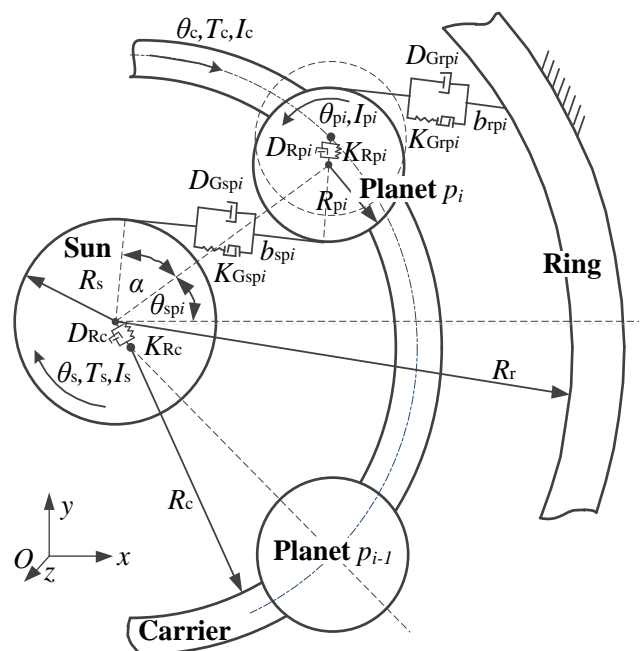

Figure 1. Dynamic model of planetary gear drive joint with multi-clearance coupling.

In Figure 1, there are $\mathrm{n}_{p}$ number of planetary gears. Each gear body $j\left(j=\mathrm{s}, \mathrm{p}_{i}, \mathrm{c}\right)$ is modeled as a rigid gear radius $R_{j}$, angular displacement $\theta_{j}$, and mass moment of inertia $I_{j}$. External torques $T_{\mathrm{s}}$ and $T_{\mathrm{c}}$ represent the input and output values. Between the planet gear $\mathrm{p}_{i}$ and gear $\mathrm{n}$ (s or $\mathrm{r}$ ), $K_{\mathrm{Gnp} i}, \mathrm{D}_{\mathrm{Gnp} i}$ and $\mathrm{b}_{\mathrm{np} i}$ are the periodically time-varying meshing stiffness, the viscous damper coefficient and backlash, respectively. $\alpha$ is theoretically pressure angle. $K_{\mathrm{Rp} i}$ and $D_{\mathrm{Rp} i}$ are the nonlinear stiffness and damping of the radial contact collision model at the planet gear bearing, respectively. $\theta_{\mathrm{sp} i}$ is the initial phase angle of planet gear relative to sun gear. And $R_{\mathrm{r}}$ is radius of ring gear. $K_{\mathrm{Rc}}$ and $D_{\mathrm{Rc}}$ are the nonlinear stiffness and damping of carrier output shaft and bearing radial contact collision model, respectively.

The equations of motion of planetary system are written as follows:

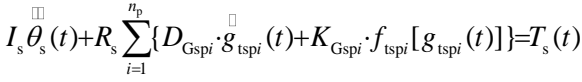

$$
\begin{aligned}
& I_{\mathrm{cp}} \theta_{\mathrm{c}}^{\mathbb{1}}(t)+I_{\mathrm{p}} \sum_{i=1}^{n_{\mathrm{p}}} \theta_{\mathrm{cpi}}^{\mathbb{W}}(t)-R_{\mathrm{s}} \sum_{i=1}^{n_{\mathrm{p}}}\left\{D_{\mathrm{Gspi} i} \cdot g_{\mathrm{tspi}}(t)+K_{\mathrm{Gspi}} \cdot f_{\mathrm{tsp} i}\left[g_{\mathrm{tspi}}(t)\right]\right\} \\
& -R_{\mathrm{r}} \sum_{i=1}^{n_{\mathrm{p}}}\left\{D_{\text {Grpi }} \cdot g_{\text {trpi }}(t)+K_{\text {Grpi }} \cdot f_{\text {trpi }}\left[g_{\text {trpi }}(t)\right]\right\}=T_{\mathrm{c}}(t) \\
& m_{\mathrm{c}} \delta_{\mathrm{cx}}(t)+\sum_{i=1}^{n_{\mathrm{p}}}\left\{D_{\mathrm{Rpi}} \cdot \delta_{\mathrm{p} i x}(t)+K_{\mathrm{Rp} i} \cdot f_{\mathrm{rp} i x}\left[\delta_{\mathrm{p} i x}(t)\right]\right\}-D_{\mathrm{Rc}} \cdot \delta_{\mathrm{c} x}(t) \\
& -K_{\mathrm{Rc}} \cdot f_{\mathrm{rcx}}\left[\delta_{\mathrm{cx}}(t)\right]=0 \\
& m_{\mathrm{c}} \delta_{\mathrm{cy}}(t)+\sum_{i=1}^{n_{\mathrm{p}}}\left\{D_{\mathrm{Rp} i} \cdot \delta_{\mathrm{p} i y}(t)+K_{\mathrm{Rp} i} \cdot f_{\mathrm{rp} i y}\left[\delta_{\mathrm{p} i y}(t)\right]\right\}-D_{\mathrm{Rc}} \cdot \delta_{\mathrm{cy}}(t) \\
& -K_{\mathrm{Rc}} \cdot f_{\mathrm{rcy}}\left[\delta_{\mathrm{cy}}(t)\right]=0
\end{aligned}
$$

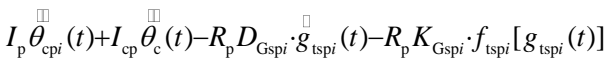

$$
\begin{aligned}
& +R_{\mathrm{p}} D_{\text {Grpi }} \cdot g_{\text {trpi }}(t)+R_{\mathrm{p}} K_{\text {Grpi }} \cdot f_{\text {trpi }}\left[g_{\text {trpi }}(t)\right]=0 \\
& m_{\mathrm{pi}} \delta_{\mathrm{p} i x}(t)-D_{\mathrm{Rp} i} \cdot \delta_{\mathrm{p} i x}(t)-K_{\mathrm{Rp} i} \cdot f_{\mathrm{rppix}}\left[\delta_{\mathrm{pix}}(t)\right]=0 \\
& m_{\mathrm{p} i} \delta_{\mathrm{p} i y}(t)-D_{\mathrm{Rp} i} \cdot \delta_{\mathrm{p} i y}(t)-K_{\mathrm{Rp} i} \cdot f_{\mathrm{rpiy}}\left[\delta_{\mathrm{p} i y}(t)\right]=0
\end{aligned}
$$

In these equations, $i=1,2, \ldots, n_{\mathrm{p}}$, and in Figure 1 , $\theta_{\mathrm{cp} i}(\mathrm{t})=\theta_{\mathrm{p} i}(\mathrm{t})-\theta_{\mathrm{c}}(\mathrm{t})$ represents rotation angle of planet gear with respect to carrier. Here, $I_{\mathrm{cp}}=I_{\mathrm{c}}+n_{\mathrm{p}}\left(I_{\mathrm{p}}+R_{\mathrm{c}}{ }^{2} \mathrm{~m}_{\mathrm{p}}\right)$ is equivalent mass moment of inertia of carrier.

And in these equations, the radial collision stiffness $K_{\mathrm{R} k}\left(k=c, p_{i}\right)$ could be obtained by collision experiment of two spheres, it is defined as:

$$
K_{\mathrm{R} k}=\frac{4}{3 \pi\left(\frac{1-v_{\mathrm{b} k}^{2}}{\pi E_{\mathrm{b} k}}+\frac{1-v_{\mathrm{j} k}^{2}}{\pi E_{\mathrm{j} k}}\right)}\left[\frac{R_{\mathrm{b} k} R_{\mathrm{j} k}}{R_{\mathrm{b} k}-R_{\mathrm{j} k}}\right]^{1 / 2}
$$

Where, $v_{i k}$ and $E_{\mathrm{i} k}(i=b, j)$ are Poisson's ratio and elastic modulus of center element $i$, respectively. The variable $\mathrm{b}$ represents bearing, and $\mathrm{j}$ represents shaft.

The damper coefficients of radial collision, namely, $D_{\mathrm{Rc}}$ and $D_{\mathrm{Rp} i}$ are defined as

$$
D_{\mathrm{R} k}=\frac{3 K_{\mathrm{R} k}\left(1-c_{e k}^{2}\right) \delta_{k}^{m}}{4 \delta_{k}^{(-)}}
$$

Where $c_{e k}$ is coefficient of restitution, $\dot{\delta}_{k}^{(-)}$is initial relative velocity at the collision location.

The time-varying meshing stiffness $K_{\mathrm{G} h} \quad(h=\mathrm{sp} i$, $\mathrm{rp} i$ )between gear teeth is given by

$$
K_{\mathrm{G} h}(t)=k_{\mathrm{Gm} k}+k_{\mathrm{G} a} \cos \left(\omega_{\mathrm{G} h} t+\varphi_{\mathrm{G} h}\right)
$$

Where, $k_{\mathrm{Gm} h}$ is average meshing stiffness, $k_{\mathrm{Ga} h}$ is time-varying meshing stiffness, $\omega_{\mathrm{G} h}$ is gear meshing frequency. $\varphi_{\mathrm{G} h}$ is initial phase of variable stiffness $\left(\varphi_{\mathrm{G} h}=0\right.$ in general ).

$D_{\mathrm{Gsp} i}$ and $D_{\mathrm{Grp} i}$ are the non-linear damper of gear meshing. In order to prevent the discontinuity of damping force during the meshing process and avoid the phenomenon in which the impact force of linear damping model is not equals to zero in critical contact state, the nonlinear damping force can be expressed as

$$
D_{\mathrm{G} h}=\left\{\begin{array}{lr}
D_{\mathrm{Gm} h} & f_{\mathrm{g} h}\left(g_{\mathrm{t} h}\right) \geq d_{h} \\
D_{\mathrm{G} m h}-\beta_{h}^{2}\left(3-2 \beta_{h}\right) & 0 \leq f_{\mathrm{g} h}\left(g_{\mathrm{th}}\right)<d_{h} \\
0 & f_{\mathrm{g} h}\left(g_{\mathrm{th}}\right)<0
\end{array}\right.
$$

Where $D_{\mathrm{Gm} h}$ is the maximum damping coefficient; $d_{h}$ is the maximum embedding depth and values as $0.1 \mathrm{~mm}$, and $\beta_{h}$ is defined as $\beta_{h}=f_{\mathrm{g} h}\left(g_{\mathrm{t} h}\right) / d_{h}$.

\subsection{Dynamic Model of Dual-Axis Drive Mechanism of Satellite Antenna with Multi-Clearance Coupling}

The dual-axis drive mechanism of satellite antenna, which be used in the inter-satellite link, is a typical multi-joint aerospace mechanism. It consists of azimuth axis and pitch axis, and the two axes are perpendicular to each other as shown in Figure 2.

In the current study, the driving mechanism of azimuth and pitch axis joints were converted into two plane rotation hinge, respectively. The model only considers the influence of radial clearance on the mechanism. And the kinetic equation is shown in equation (6): 


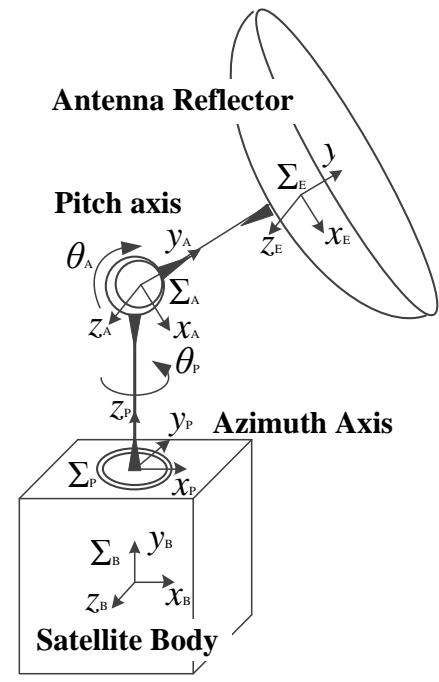

Figure 2. Dynamic model of dual-axis drive mechanism of satellite antenna with multi-clearance coupling.

$$
\left\{\begin{array}{l}
\boldsymbol{M} \ddot{\boldsymbol{q}}+\boldsymbol{C} \dot{\boldsymbol{q}}+\boldsymbol{K} \boldsymbol{q}+\boldsymbol{\Phi}_{q}^{T} \lambda=\boldsymbol{F} \\
\boldsymbol{\Phi}(\boldsymbol{q}, t)=0
\end{array}\right.
$$

Where $\boldsymbol{q}$ is a generalized coordinate array, $\boldsymbol{M}$ is a generalized mass matrix of the gear rotor system, $\boldsymbol{C}$ and $\boldsymbol{\Phi}_{q}^{T}$ are the damping matrix and jacobian of the constaints, respectively. $\boldsymbol{K}$ is a generalized stiffness matrix, $\lambda$ refer to Lagrangian Multiplier vector, $\boldsymbol{F}$ is a generalized external force matrix.

Because a coupling relationship between radial clearance and backlash is existed. So after considering the multi-clearance coupling in the dynamic model of double-axis drive mechanism, the kinetic equation becomes as shown in equation (7):

$$
\boldsymbol{M}_{p} \ddot{\boldsymbol{q}}_{p}=\boldsymbol{Q}_{F}+\boldsymbol{Q}_{V}+\boldsymbol{Q}_{C}
$$

Where $\boldsymbol{M}_{p}$ is a generalized mass matrix of all bodies in the double-axis drive mechanism (including the internal structure of a planetary gear drive joint), $\boldsymbol{q}_{p}$ is a generalized coordinate array. $\boldsymbol{Q}_{\boldsymbol{F}}$ and $\boldsymbol{Q}_{\boldsymbol{V}}$ are generalized external force matrix and the quadratic term related to velocity, respectively. And $\boldsymbol{Q}_{C}$ is the internal force matrix of the system at the clearance. Take into account the forces and moments inside the planetary gear joint, including the dynamic meshing torque $T_{\mathrm{sp} i}$ between the sun gear and planetary gear $i$, the dynamic meshing torque $T_{\text {rp } i}$ between planetary gear $i$ and ring gear, the radial contact force between the planetary gear and the planet carrier is $F_{\mathrm{Rp} i}$, and the radial contact force between the output axis of the planet carrier and the axle sleeve is $F_{\mathrm{R} c}$, the equations are expressed as:

$$
\begin{aligned}
& {\left[\begin{array}{ll}
\boldsymbol{m}_{R R} & \boldsymbol{m}_{R \theta} \\
\boldsymbol{m}_{R \theta} & \boldsymbol{m}_{\theta \theta}
\end{array}\right] \cdot\left[\begin{array}{c}
\ddot{\boldsymbol{R}} \\
\ddot{\boldsymbol{P}}
\end{array}\right]=\left[\begin{array}{l}
\boldsymbol{Q}_{F R} \\
\boldsymbol{Q}_{F \theta}
\end{array}\right]+\left[\begin{array}{l}
\boldsymbol{Q}_{V R} \\
\boldsymbol{Q}_{V \theta}
\end{array}\right]} \\
& +\left[\begin{array}{c}
\sum_{j=1}^{2}\left(\begin{array}{c}
\left.\sum_{i=1}^{n_{p}} \boldsymbol{Q}_{C R}\left(T_{\mathrm{sp} i j}\right)+\sum_{i=1}^{n_{p}} \boldsymbol{Q}_{C R}\left(T_{\mathrm{rp} i j}\right)\right) \\
+\sum_{i=1}^{n_{p}} \boldsymbol{Q}_{C R}\left(F_{\mathrm{Rp} i j}\right)+\boldsymbol{Q}_{C R}\left(F_{\mathrm{Rcj}}\right)
\end{array}\right) \\
\sum_{j=1}^{2}\left(\begin{array}{c}
\left.\sum_{i=1}^{n_{p}} \boldsymbol{Q}_{C \theta}\left(T_{\mathrm{sp} i j}\right)+\sum_{i=1}^{n_{p}} \boldsymbol{Q}_{C \theta}\left(T_{\mathrm{rp} i j}\right)\right) \\
+\sum_{i=1}^{n_{p}} \boldsymbol{Q}_{C \theta}\left(F_{\mathrm{Rp} i j}\right)+\boldsymbol{Q}_{C \theta}\left(F_{\mathrm{Rcj}}\right)
\end{array}\right)
\end{array}\right]
\end{aligned}
$$

Where, $\boldsymbol{m}_{R R}$ is the mass matrix of translational part of all bodies in the system generalized mass matrix. $\boldsymbol{R}$ and $\boldsymbol{P}$ are the position vector and the quaternion of all the bodies in the inertial coordinate system, respectively. And $\boldsymbol{m}_{\theta \theta}$ is the inertial tensor of all the body rotating parts. $\boldsymbol{Q}_{F R}$ and $\boldsymbol{Q}_{\boldsymbol{F} \theta}$ are the generalized forces of the external forces corresponding to translation and rotation respectively. $\boldsymbol{m}_{R \theta}$ means the inertial coupling of all body moving and rotating, and $\boldsymbol{Q}_{\boldsymbol{V} \boldsymbol{R}}$ and $\boldsymbol{Q}_{\boldsymbol{V} \theta}$ are the velocity dependent quadratic terms corresponding to translation and rotation, respectively.

\section{Numerical simulation}

In Figure 2, the azimuth axis is connected to the satellite body, which drives the entire drive mechanism and antenna reflector rotation during the movement, and the pitch axis is located between the azimuth axis and antenna reflector. Above all, the movement of azimuth axis and pitch axis not only affect each other, but also affect the dynamics positioning accuracy of antenna reflector. In this section, the dynamic response of antenna reflector of dual-axis drive mechanism with multi-clearance is studied for the simultaneous rotation of dual-axis at different rotation speeds.

In actual work, the dual-axis drive mechanism exists in which two axis rotate at the same time. But from the control point of view, the azimuth and pitch axis does not have a larger rotational speed difference, namely, the rotational speed of the azimuth and pitch axis is equal. Here, three typical joint output speeds are selected, that is, $0.49 \%, 0.98 \%$, and $1.96 \%$ s. And the radial clearance and backlash in the dual-axis transmission joints are taken as $100 \mu \mathrm{m}$.

Figure 3 shows the positional error curves of satellite antenna reflector on the three directions $\mathrm{X}, \mathrm{Y}$ and $\mathrm{Z}$ axis at three typical rotational speeds. It can be see from in Figure 2 that the positional error curves of satellite antenna reflector in the direction $\mathrm{X}$ axis is large, while in the direction $\mathrm{Y}$ and $\mathrm{Z}$ axis is nearly equal to each other. The main reason is that azimuth and pitch axis are perpendicular to each other, which leads to the position error superposition in the direction $\mathrm{X}$ axis. By comparing curve (a), (b) and (c) in Figure 3, it can be see that the amplitude of position error of $\mathrm{X}$ axis's direction is increases with an increase in rotational speed. This phenomenon is mainly due to the rotation speed increases, the output shaft of dual-axis and the sleeve is in state of 
continuous contact, as the collision contact deformation increases, the positional error of antenna reflector also fluctuates greatly during the movement.

Figure 4 shows rotational speed error curves of satellite antenna reflector on the three directions $\mathrm{X}, \mathrm{Y}$ and $\mathrm{Z}$ axis at three typical rotational speeds. It is noteworthy

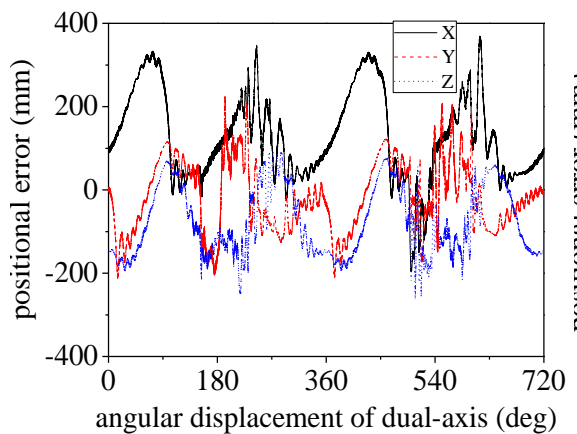

(a) Rotational speed $0.49 \%$

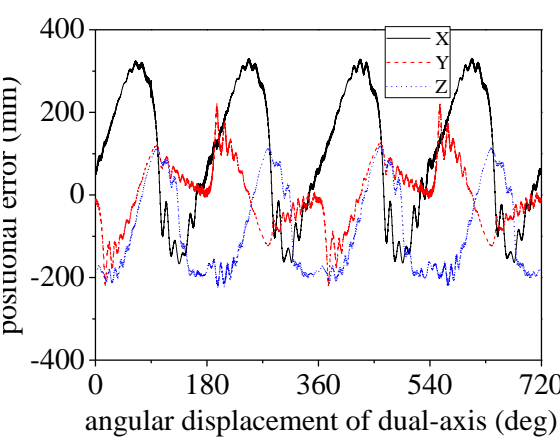

(b) Rotational speed $0.98 \%$

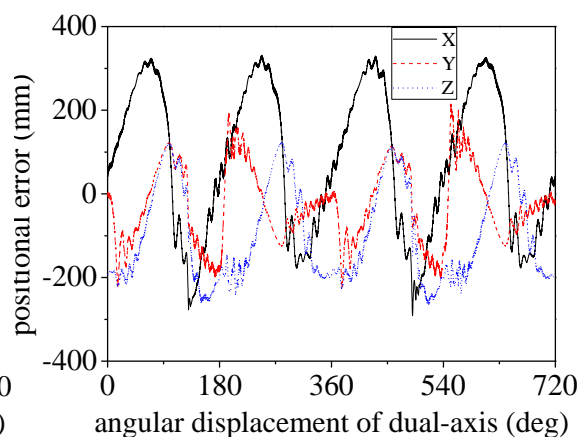

(c) Rotational speed $1.96 \%$

Figure 3. Positional error curves of antenna reflector at different rotational speed.

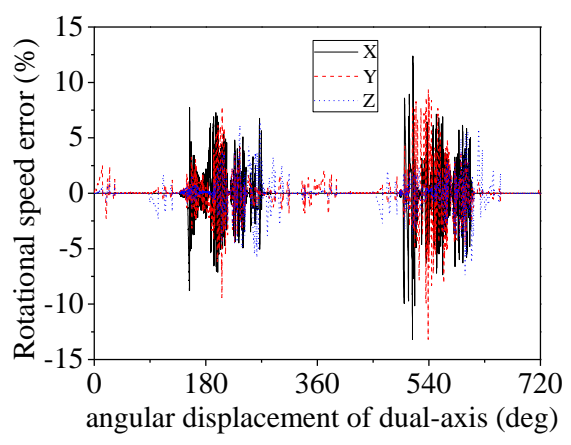

(a) Rotational speed $0.49 \%$

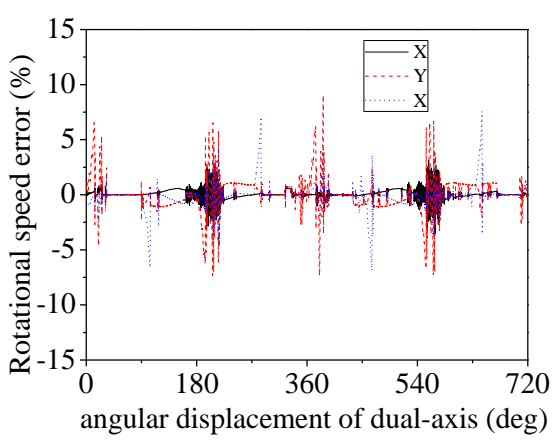

(b) Rotational speed $0.98 \%$

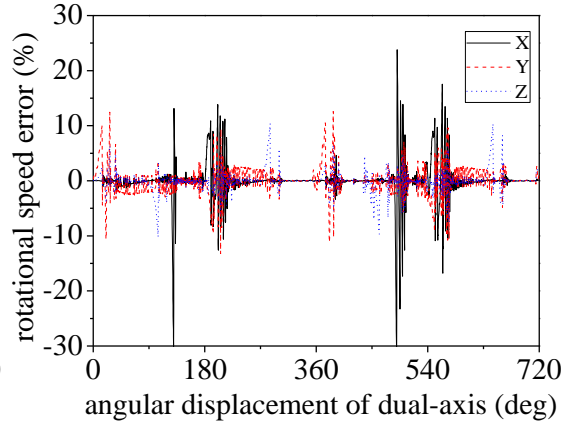

(c) Rotational speed $1.96 \%$

Figure 4. Rotational speed error curves of antenna reflector at different rotational speed.

relatively small at the rotational speed of $0.98 \%$ s, and the stability of system is better. However, when the rotational speed is $0.49 \%$, the rotational speed error of satellite antenna reflector is not reduced with the lower rotational speed. And the amplitude of rotational speed error also fluctuates considerably at the rotational speed of $1.96 \%$ s.

\section{Conclusions}

This analytical study on dynamic positioning accuracy of dual-axis drive mechanism of satellite antenna with multi-clearance coupling. First, this paper sets up a new nonlinear dynamic model of planetary gear joint, in which the radial clearance of bearing, dynamic backlash and time-varying meshing stiffness are considered. Second, the dynamic model of double-axis driving mechanism of satellite antenna with multi-clearance coupling is established. Finally, the effects of multi-clearance coupling on positional and rotational speed error curves of antenna reflector at different rotational speed are studied and discussed respectively by numerical simulation. It can be found that the amplitude of position error of $X$ axis's direction is increases with an increase in rotational speed, while in the direction $\mathrm{Y}$ and $\mathrm{Z}$ axis is nearly equal to each other. For rotational speed errors curves of antenna reflector, the amplitude in the three axial directions are relatively small at the rotational speed of $0.98 \%$, and the stability of system is better.

\section{Acknowledgment}

This manuscript is funded by Science and Technology on Space Intelligent Control Laboratory, No.ZDSYS-2017 -08, and State Key Laboratory of Robotics and System (HIT), No.SKLRS-2017-KF-15, the National Natural Science Foundation of China (NSFC), No.51575126, and the Graduate Innovative Ability Training Project of Hebei Province, NO.CXZZSS2018025. The authors would like to thank the research grant from Spacecraft Dynamics Design and Simulation Lab (SDDSL) of HIT.

\section{References}

1. E. Salahshoor, S. Ebrahimi, M. Maasoomi. Mech. Mach. Theory. 105 495-509 (2016)

2. H. Zhang, J. Tian, J. Zhou, Y. Zhao, B. You. Chin. J. Mech. Eng. 53 29-37 (2017)

3. A. Alshyyab, A. Kahraman. Proc. Inst. Mech. Eng. K. J. Multi-Body Dyn. 221 567-576 (2007)

4. P. Sondkar, A. Kahraman. Mech. Mach. Theory. 70 157-174 (2013)

5. M. R. Kang, A. Kahraman. J. Sound. Vib. 350 11-29 (2015) 
6. M. Filipe, I. Fernando, D. Nuno, F. Paulo. Mech. Mach. Theory. 116 123-144 (2017)

7. C. G. Cooley, R. G. Parker. Mech. Syst. Signal. Process. 40 91-104 (2013)

8. Y.Guo, T. Eritenel, T. M. Ericson, R. G. Parker. J. Sound. Vib. 333 5762-57852014

9. X.Dai, C. G. Cooley, R. G. Parker. Mech. Mach. Theory. 101 60-74 (2016)

10. B. Pan, D. Yu, J. Sun. J. Asrton. 31 2448-55 (2010) 